\title{
Gut feeling on bacterial GPCR agonists
}

The mechanisms through which microorganisms in the gut affect human health are poorly defined. Writing in Nature, Cohen and colleagues describe commensal bacterial metabolites that activate $\mathrm{G}$ protein-coupled receptors (GPCRs) in the intestine, including GPR119, thereby improving glucose tolerance in mice.

In previous work, this group had identified a human microbiotaencoded, GPCR-active long-chain $\mathrm{N}$-acyl amide called commendamide. Similar molecules, such as the endocannabinoids, are known GPCR modulators. In their new article, they searched the Human Microbiome Project database for genes encoding $\mathrm{N}$-acyl synthases (NASs) as a source of potential GPCR modulators,

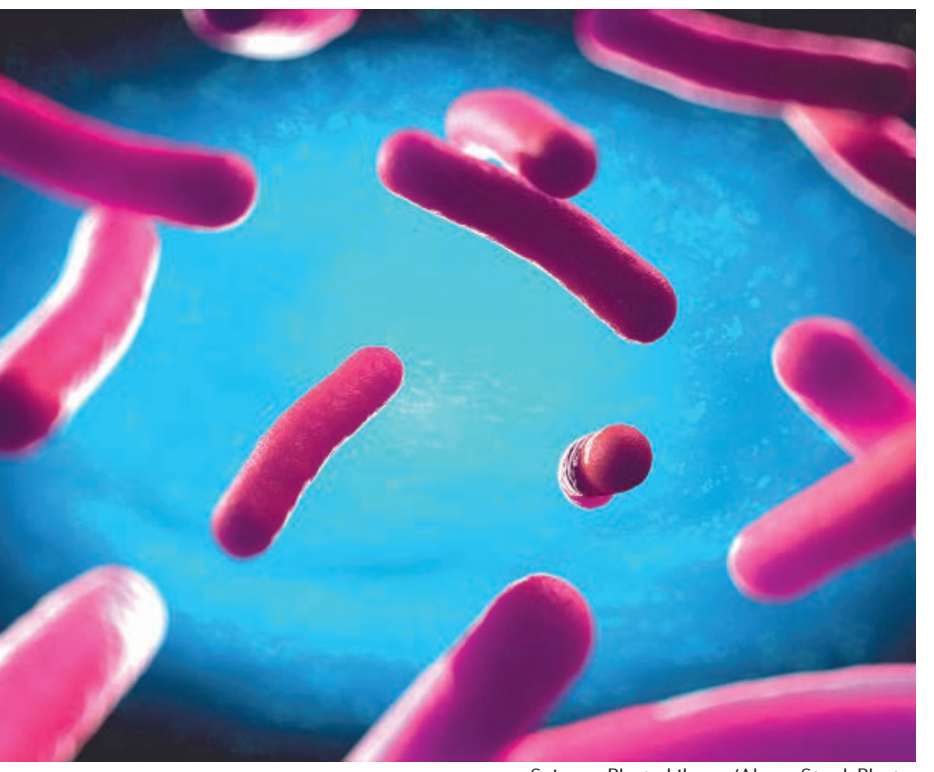

Science Photo Library/Alamy Stock Photo identified 143 genes, and cloned and expressed 44 of them in bacteria (chosen to maximize diversity). Thirty-one metabolites were identified from these clones, which could be grouped into six families, differing by amine group and fatty acid tail.

Despite interindividual variation, these NAS genes were prevalent in gastrointestinal bacteria from human samples, and different clades could be found in various parts of the gastrointestinal tract. Site-specific expression of the NAS-encoding genes in existing metatranscriptome sequence data from stool and supragingival plaque microbiomes supported the hypothesis of site-specific $N$-acyl amide production.

The six $N$-acyl amides were then used to probe a library of GPCRs to identify molecule-receptor pairs. $N$-palmitoyl serinol potently activated GPR119, N-3-hydroxypalmitoyl ornithine activated sphingosine-1phosphate receptor $4\left(\mathrm{~S}_{1} \mathrm{P}_{4} \mathrm{R}\right)$, $\mathrm{N}$-myristol alanine activated GPR132 and $N$-acyloxyacyl glutamine inhibited two prostaglandin receptors. These receptors have diverse roles in human biology: GPR119 regulates glucose homeostasis, in part through the release of insulin and glucagon-like peptide 1 (GLP1); $\mathrm{S}_{1} \mathrm{P}_{4} \mathrm{R}$ controls immune functions and the prostaglandin receptors have numerous roles depending on tissue expression and ligand.

Relative to the known endogenous ligands, the bacterially encoded ligands for GPR119 and $\mathrm{S}_{1} \mathrm{P}_{4} \mathrm{R}$ had similar potency, but greater maximum activation (GPR119) or greater specificity $\left(\mathrm{S}_{1} \mathrm{P}_{4} \mathrm{R}\right)$ in in vitro assays. In mouse enteroendocrine cells, the endogenous and bacterially encoded ligands induced GLP1 secretion to similar levels.

Given that the metabolic effects of the endogenous GPR119 agonist oleoylethanolamide are thought to occur through interactions in the intestinal mucosa, as intravenous delivery fails to elicit a response, the authors therefore investigated whether colonization of mice with bacteria that had been engineered to produce $\mathrm{N}$-acyl serinols would alter blood glucose levels. Indeed, blood glucose levels in mice colonized with these engineered bacteria peaked at a lower level following oral glucose administration than did blood glucose levels in mice colonized with wild-type bacteria. GLP1 and insulin levels were upregulated in the treated mice relative to controls.

This work provides a potential mechanistic link between commensal gut bacteria and metabolism, immune cell differentiation and trafficking and tissue repair. Targeting these receptors or $\mathrm{N}$-acyl amides could potentially be used to therapeutically alter this interaction.

Megan Cully

ORIGINAL ARTICLE Cohen, L. J. et al. Commensal bacteria make GPCR ligands that mimic human signalling molecules. Nature 549, 48-53 (2017) FURTHER READING Oh, D. Y \& Olefsky, J. M. G protein-coupled receptors as targets for anti-diabetic therapeutics. Nat. Rev. Drug Discov. 15, 161-172 (2016) 